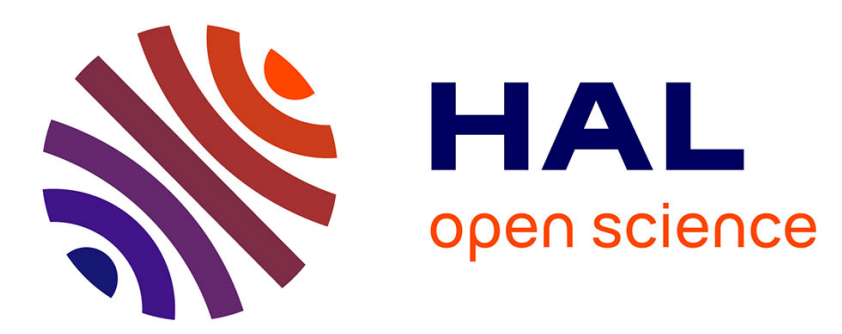

\title{
Non Linear Effects Produced by Electrostatic Waves in a Magnetized Plasma
}

C. Riccardi, A. Penso, D. Xuantong, M. Fontanesi

\section{To cite this version:}

C. Riccardi, A. Penso, D. Xuantong, M. Fontanesi. Non Linear Effects Produced by Electrostatic Waves in a Magnetized Plasma. Journal de Physique IV Proceedings, 1995, 05 (C6), pp.C6-85-C6-88. 10.1051/jp4:1995616 . jpa-00253979

\section{HAL Id: jpa-00253979 https://hal.science/jpa-00253979}

Submitted on 1 Jan 1995

HAL is a multi-disciplinary open access archive for the deposit and dissemination of scientific research documents, whether they are published or not. The documents may come from teaching and research institutions in France or abroad, or from public or private research centers.
L'archive ouverte pluridisciplinaire HAL, est destinée au dépôt et à la diffusion de documents scientifiques de niveau recherche, publiés ou non, émanant des établissements d'enseignement et de recherche français ou étrangers, des laboratoires publics ou privés. 


\title{
Non Linear Effects Produced by Electrostatic Waves in a Magnetized Plasma
}

\author{
C. Riccardi, A. Penso, D. Xuantong* and M. Fontanesi
}

Dipartimento di Fisica, Università degli Studi di Milano, Via Celoria, 16 - 20133 Milano, Italy

* Istituto di Fisica del Plasma, CNR, Via Bassini, 15 - 20133 Milano, Italy

\begin{abstract}
Some non-linear phenomena, like ponderomotive effects and parametric decays, produced by high power electrostatic waves in a magnetized low density plasma, have been investigated experimentally. The injection of high power electrostatic waves into a plasma causes ponderomotive forces arise. Their action primarily gives rise to a plasma density depletion in front of the launching system and subsequently affects both the wave injection and propagation, producing cavitons.

Beside these effects, RF power produces parametric decays which are also responsible of an edge electron heating.

Above mentioned phenomena were studied in the range of low frequencies $(1 \div 100 \mathrm{MHz})$, in conditions of high $\left(n_{e} \approx 10^{11} \mathrm{~cm}^{-3}\right)$ and low $\left(n_{e} \approx 10^{9} \mathrm{~cm}^{-3}\right)$ density, analyzing the modifications of the electron density and temperature profiles, the shape of e.s. waves's electric field and the spectrum of the detected signals.
\end{abstract}

\section{GENERAL DEDUCTION OF A PONDEROMOTIVE FORCE DENSITY}

To interpret accurately the ponderomotive effects, it is necessary to include the ponderomotive force in the fluid description of plasmas by calculating the generalized force density term $\nabla \cdot(n m \underline{v} \underline{v})$ in a non-collisional momentum balance equation

$$
\frac{\partial}{\partial t}(n m \underline{v})+\nabla \cdot(n m \underline{v} \underline{v})+\nabla \cdot \underline{p}+n e\left(\underline{E}+\frac{1}{c} \underline{v} \times \underline{B}\right)=0
$$

and defining

$$
\underline{F}_{\mathrm{PM}}=\frac{1}{n(\underline{r})} \underline{f}_{\mathrm{PM}}(\underline{r})=\frac{-1}{n(\underline{r})}\langle\nabla \cdot n m \underline{v} \underline{v}\rangle_{T}=-\nabla V_{\mathrm{PM}}
$$

where $n(r)$ is the particle number density and $\langle\ldots\rangle_{T}$ is the average value in an oscillation period of the particle motion.

One obtains, in slab geometry: 


$$
\begin{aligned}
\underline{f}_{\mathrm{PM}}=-\frac{1}{8 \pi} \sum_{s}\left\{\underline { \hat { x } } \left[\frac{\partial}{\partial x}\left(\frac{\omega_{p s}^{2} \omega^{2}}{\left(\omega^{2}-\Omega_{s}^{2}\right)^{2}} E_{\perp}^{2}\right)\right.\right. & \left.+\frac{\partial}{\partial z}\left(\frac{\omega_{p s}^{2}}{\omega^{2}-\Omega_{s}^{2}} E_{\perp} E_{\|}\right)\right] \\
& \left.+\underline{\hat{z}}\left[\frac{\partial}{\partial x}\left(\frac{\omega_{p s}^{2}}{\omega^{2}-\Omega_{s}^{2}} E_{\perp} E_{\|}\right)+\frac{\partial}{\partial z}\left(\frac{\omega_{p s}^{2}}{\omega^{2}} E_{\|}^{2}\right)\right]\right\}
\end{aligned}
$$

where $\omega_{p s}$ and $\Omega_{s}$ are the plasma and the cyclotron frequencies of that species of particles.

In the WKB approximation, one can simplify eq. 3 by expressing the derivatives of the electric field intensity, obtaining a general form of the ponderomotive force density that include terms which take into account the ion dynamics and forces due to density gradients:

$$
\underline{f}_{\mathrm{PM}}=-\frac{1}{8 \pi} \sum_{s}\left\{\hat{\hat{x}} \frac{\partial}{\partial x}\left[\left(\frac{\omega_{p s} \omega}{\omega^{2}-\Omega_{s}^{2}}\right)^{2} E_{\perp}^{2}+\frac{\omega_{p s}^{2}}{\omega^{2}-\Omega_{s}^{2}} E_{\|}^{2}\right]+\underline{\underline{z}} \frac{\partial}{\partial z}\left[\frac{\omega_{p s}^{2}}{\omega^{2}} E_{\|}^{2}+\frac{\omega_{p s}^{2}}{\omega^{2}-\Omega_{s}^{2}} E_{\perp}^{2}\right]\right\} \text {. }
$$

Eq.4 introduces ponderomotive effects due to density gradients through the derivatives of $\omega_{p s}$.

If $\underline{f}_{\mathrm{PM}}$ is the ponderomotive force density on the plasma fluid, the ponderomotive potential $V_{\mathrm{PM}}$ is its integral in space. To write down a simple form of $V_{\mathrm{PM}}$ we shall consider high frequencies. Far from the resonance with $\Omega_{s}$ (this means $\omega \gg \Omega_{s}$ ) one can simply write:

$$
V_{\mathrm{PM}}=\sum_{s} \frac{1}{n_{s}} \frac{\omega_{p s}^{2}}{\omega^{2}} \frac{E^{2}}{8 \pi}=\sum_{s} \frac{e^{2} E^{2}}{2 m_{s} \omega^{2}}
$$

In both the IBW and the EPW regimes, near the lower hybrid resonance, the perpendicular wave electric field dominates the parallel one $\left(E_{\perp} \gg E_{\|}\right)$because of the fact that $k_{\perp} \gg k_{\|}$ $\left(E_{\perp} / E_{\|}=k_{\perp} / k_{\|} \approx 10^{2}\right)$. The ponderomotive force can be then approximated neglecting the $z$ component in eq. 4 and considering only the term depending on $E_{\perp}$. It turns out that the ponderomotive force acts perpendicularly to the magnetic field, pushing, in front of the antennas, the plasma edge to the inside:

$$
\underline{f}_{\mathrm{PM}}=\hat{x} \frac{\partial}{\partial x}\left(\frac{\omega_{p i}^{2} \omega^{2}}{\left(\omega^{2}-\Omega_{i}^{2}\right)^{2}} \frac{E_{\perp}^{2}}{4 \pi}\right),
$$

where we neglected all electronic terms because of the low density values in our experiment. In fact to match the ionic terms of eq. $6, \omega_{p e}^{2} / \omega^{2}$ (eq.4) has to grow to about $10^{4}$. At relatively high densities (about $10^{10} \mathrm{~cm}^{-3}$ ) the parallel action of the ponderomotive forces, mainly supported by electrons, again becomes significant and comparable to the perpendicular ionic term. 


\section{EXPERIMENTAL RESULTS}

The experiment is performed on the Thorello device which has the following parameters: major radius $R=40 \mathrm{~cm}$, minor radius $a=8 \mathrm{~cm}$, toroidal magnetic field $B_{o}=2.3 \mathrm{kG}$, plasma density $n=10^{8} \div 10^{11} \mathrm{~cm}^{-3}$, electron temperature $T_{e}=1 \div 10 \mathrm{eV}$, ion temperature $T_{i}=0.1 \div 1 \mathrm{eV}$. Previous theoretical considerations support our experimental results in so far the ponderomotive effect we revealed grows with wave power, which is proportional to $E^{2}$ and acts perpendicularly to the toroidal magnetic field as can be deduced from the density growth in the inner plasma. The plot of the experimental density value as a function of the applied power (fig.1) shows an exponential edge density decrease with power. Same behaviour was observed at different toroidal locations. The effect mostly appears before the layer where the conversion from a lower hybrid wave (LHW) to an ion Bernstein wave (IBW) takes place.

A Boltzmann balance equation permits to establish the way in which the electron density $n_{e}$ is affected by ponderomotive effects:

$$
\frac{n_{e}}{n_{o e}}=e^{-V_{\mathrm{PM}} / T_{e}}
$$

where $n_{o e}$ is the electron density in absence of $\mathrm{RF}$ and $T_{e}$ the electron temperature. The ponderomotive effect becomes relevant whenever its potential matches the thermodynamical energy of the electrons, i.e. when $V_{\mathrm{PM}} / T_{e}$ gets close to 1 . Such an exponential behaviour can be observed in fig.1 up to a wave power of $P \approx 400 \mathrm{~mW}$ while for higher power values the density results independent from injected power. This phenomenon is due to the electron heating effects (fig.1) that make the threshold value for ponderomotive effects increase.

The fact we did not observe any significant dependence of the ponderomotive force on the launched $k_{\|}$value (fig.2) confirms our hypothesis that the parallel term in eq.4 (containing $k_{\|}$from the $z$ derivatives of the electric field) is negligible compared to the perpendicular one.

In what concerns the wave modification, we could observe

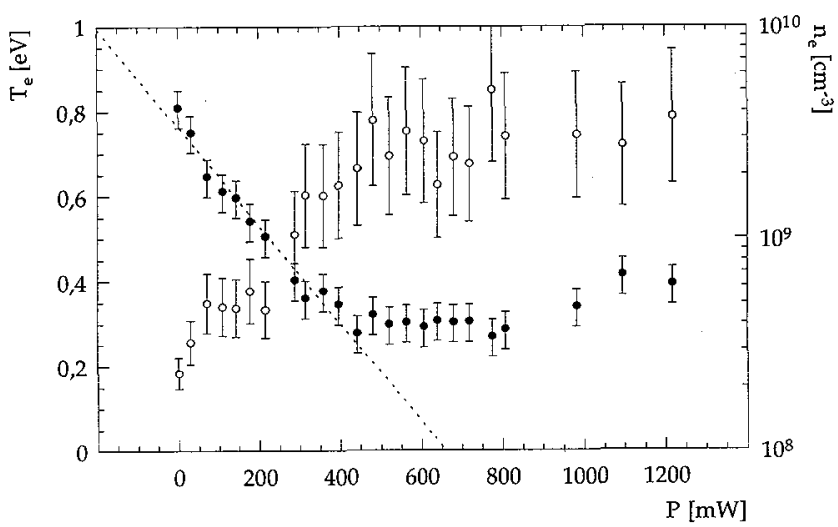

fig. 1: Dependency of electron density and electron temperature from the injected wave power for $f=6 \mathrm{MHz}, B_{0}=2 \mathrm{kG}$ how the wave envelope changed its shape while the RF-power is increased. At high power levels, the wave energy is highest where the plasma density is mostly depleted. This means that the wave is trapped in the cavity formed by ponderomotive forces and that it does not propagate to the plasma inner. 
At high frequencies the ponderomotive effect becomes less remarkable while another non-linear phenomenon arises, exspecially in experiments performed on a plasma of higher density. Fig. 3 shows parametric decay instabilities occurring for a frequency of $f=80 \mathrm{MHz}$ mainly at the plasma edge: the lower hybrid pump wave decays there into other lower hybrid waves and into ion cyclotron quasi modes. The frequencies for which this instability could be observed were in the range of $\omega \approx 20 \Omega_{i}$; the ion ciclotron modes are characterized by

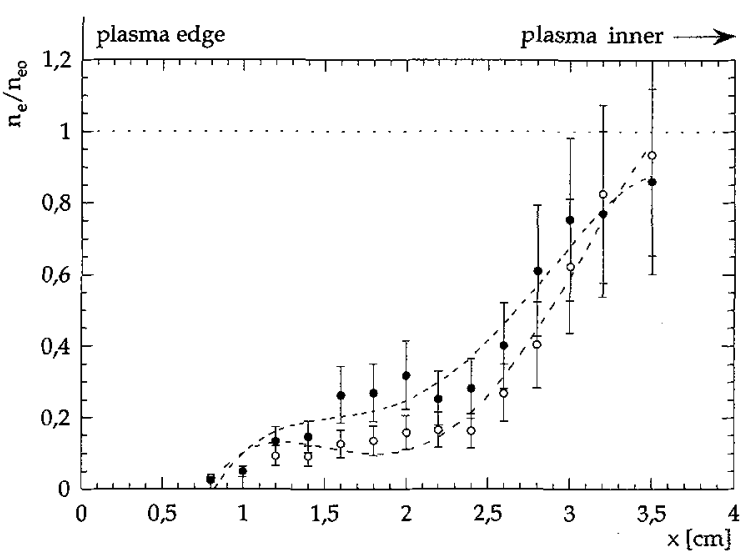

fig.2: Density profiles for two values of parallel wavevector component for $f=6 \mathrm{MHz}$ : $0: k_{\|}=0,001 \mathrm{~cm}^{-1}, \bullet: k_{\|}=0,11=\mathrm{cm}^{-1}$ frequency values near the ion ciclotron frequency $\left(\omega \approx \Omega_{i}\right)$.

The parametric decays produce a strong plasma heating at the edge so that the threshold for ponderomotive effects becomes so high that the density depletion is not observable anymore.

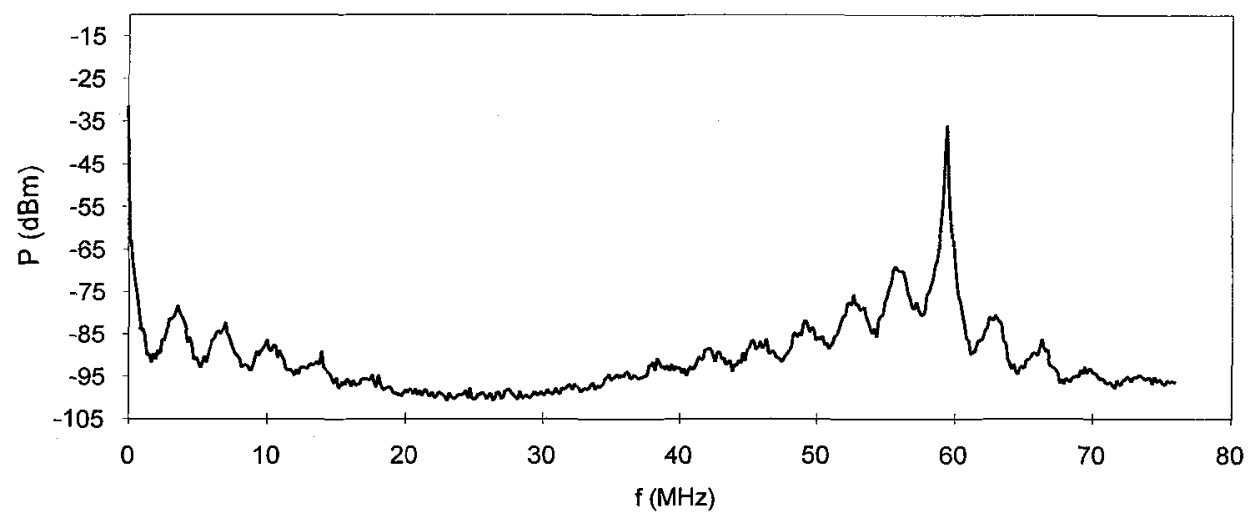

fig.3: RF signal spectrum obtained for $f=80 \mathrm{MHz}, n_{e}=10^{10} \mathrm{~cm}^{-3}, T_{e}=I \mathrm{eV}, B_{o}=2 \mathrm{kG}$

\section{REFERENCES}

[1] Wilson J.R. and Wong K.L., Phys.Fluids 25, 675 (1982)

[2] Morales G.J. and Lee Y.C., Phys.Rev.Lett. 35, 930 (1975)

[3] Pinsker R.I., Petty C.C., Mayberry M.J., Porkolab M. and Heidbrink W.W., Nucl.Fus. 33, 777 (1993)

[4] Mayberry M.J., Pinsker R.I., Petty C.C. et al., Nucl.Fus. 33, 627 (1993) 